\title{
Cloth Matching and Color Selection using Intelligent Robotic System
}

\author{
Sreema E.R ${ }^{1}$, Jisna Davis ${ }^{2}$, Jimna Rose $^{3}$, Dr. Vince Paul ${ }^{4}$ \\ Students, Department of Computer Science, Universal Engineering College, Thrissur, India ${ }^{1,2,3}$ \\ Professor, Department of Computer Science, Universal Engineering College, Thrissur, India ${ }^{4}$
}

\begin{abstract}
The system proposing is to use it in the textile area. The system incorporates with following technology, consisting of image processing and robotic arm. The technology is meant for pick up the matching cloth this will help the people to select the apparel based on color. The robot automatically recognizes cloths color proposed. The backbone technology is image processing and working under the principle of openCV. Recognized color is matched by intelligent system based on the decision. The system will pick the specific cloth based on recognized color by using robotic technology. By using this technology it reduce the error rate and increase the accuracy.
\end{abstract}

Keywords: Colour recognition, intelligent system, pick and place arm, Image processing, Robotic Technology

\section{INTRODUCTION}

Most of the people are worried about choosing matching cloths as per needed color from the textile shop. Salesman will need more time to find a suitable cloth of wanted color. So a lot of fabrics will have to be displayed to get the suitable one. Human eyes cannot continuously differentiate colors, as they get tired very soon. The system build a robot, which is capable of detecting colored cloths. It has a robotic arm, after detection of color which picks up the cloth and then place it in a predetermined place as required by the customer. In this system contains a camera along with the image processing software does the job of color detection. We have used four servomotors for rotating the arms of the robot. The arms can move horizontally from its base and vertically up and down. The gripper is capable of moving quite far for proper gripping of objects. The overall improvement in the efficiency of the production process can be significant by using this machine.

We matured a camera-based model to notice the clothing colors. The system integrates a camera \&computer. The camera captures the image of cloth given by the customer. The image will send to computer .The computer identify the color of cloth by analysing image captured. Another camera captures the image of clothes in the rack, the computer find the interested area from the image having different colors and matches the clothes as per needed color. Find the position of the cloth in the rack, send that position to arm then robotic arm pick up that cloth from the rack. Although many methods have been developed for texture matching and color detection in the computer vision and image processing research, currently there is no device that can effectively supply matching choices for people. In this system, the clothes color is detected by color detection algorithm using openCV library and matching the clothes by contrast color given by the designer. Based on color recognition detecting color of cloths, then Arduino Uno controls the servo motor of robotic arm to pick up the cloth and then place it.

\section{RELATED WORK}

${ }^{[1]}$ The paper help the visually impaired to select the apparel and independent in their decision. The application could be matches the apparel based on the dominating color. Android mobile phone is used for getting voice input from visually impaired person. Voice application was developed in the android mobile phone, which convert the voice into text and transmitted to robot through Bluetooth .The color said by the user, which is recognize by robot. Recognized color is matched by open $\mathrm{CV}$ python programming code. Then pick the clothes based on color recognition and place by the robot's pick and place arm.

${ }^{[2]}$ The project is to build a robotic sorter, which is capable of detecting and sorting colored objects. It has a robotic arm, which, after detection of color, picks up the object and then place it at a predetermined place as required by the production system. This project is a combination of electrical, mechanical and visual subsystems. A Pixy cam along with the image processing software do the job of color detection. We have used several servomotors for rotating the arms of the robot. TheArms can move horizontally from its base and vertically up and down. The gripper is capable of moving quite far for proper gripping of objects. This multi-DOF (Degree of Freedom) robotic sorter can be a very 
useful tool in fully automating a production process, which uses conveyer belts. The overall improvement in the efficiency of the production process can be significant by using this machine.

${ }^{[3]}$ Edge detection is the name for a set of mathematical methods which aim at identifying points in a digital image at which the image brightness changes sharply or, more formally, has discontinuities. The points at which image brightness changes sharply are typically organized into a set of curved line segments termed edges. The same problem of finding discontinuities in 1D signals is known as step detection and the problem of finding signal discontinuities over time is known as change detection. Edge detection is a fundamental tool in image processing, machine vision and computerVision, particularly in the areas of feature detection and feature extraction.

${ }^{[4]}$ Matching clothes is a challenging task for many blind people. In this paper, we present a proof of concept system to solve this problem. The system consists of 1) a camera connected to a computer to perform pattern and color matching process; 2) speech commands for system control and configuration; and 3) audio feedback to provide matching results for both color and patterns of clothes. This system can handle clothes in deficient color without any pattern, as well as clothing with multiple colors and complex patterns to aid both blind and color deficient people. Furthermore, our method is robust to variations of illumination, clothing rotation and wrinkling. To evaluate the proposed prototype, we collect two challenging databases including clothes without any pattern, or with multiple colors and different patterns under different conditions of lighting and rotation. Results reported here demonstrate the robustness and effectiveness of the proposed clothing matching system.

${ }^{[5]}$ In this paper, a camera based prototype system is proposed. The system is capable of recognizing the clothing pattern and color. The system consists of ARM micro controller with camera. The picture of cloth is fed as input to pattern analyser. The pattern analyser detects the color of the cloth, texture and design pattern. Speakers aid the visually impaired by playing the pre-recorded message. The proposed system is found to efficient and simple to use for the visually impaired people.

${ }^{[6]}$ Automatic vision based robots are intelligent robots which captures visual data, process it and delivers an appropriate output. These robots require less human intervention as it is prefer with instructions. An efficient approach is used to capture and process the images. Open source computer vision (Open CV) is used for real time capturing the image and Raspberry Pi for its processing. Image is captured on the bases of Hue, saturation and color value range. Numerous Open CV library functions are used for loading the image, framing the image and differentiating images on the basis of HSV value. The aim is to construct a model that can recognize sign boards and take appropriate path leading towards the destination.

\section{PROPOSED SYSTEM}

The system is camera based prototypesystem.In this system, the clothes color is detected by color detection algorithm using open CV library and matching the clothes by contrast color given by the designer. The camera captures the image of cloth and matches the cloth as per information given in the C++ code. Computer track the location of the cloth and the pick and place arm picks the cloth as per the instruction given by Arduino Uno.

The captured image is processed by image processing technique. The image of cloth is analyzed for various colors like Red, blue etc. The software is programmed to recognize these colors. Colors are detected and identified, for example the captured image has red color then red color is detected and Some 15 to 20 colors can be detected approximately. The detection of color depends on camera resolution and lighting effects and after detection of cloth color, the cloths is picking up by the arm of the robot and places it into desired location.

This system contain two parts, a pick and place arm and image processing. The pick and place arm contains servomotors and a camera. The Arduino Uno controls the movement of arm. A computer is used for image processing. A camera placed in front of the rack which contains the cloth. The customer shows the color of the cloth to the robotic system, the camera on the arm capture the image, analyse the color from the frame using color thresholding. Second camera capture the picture and analyse the color which match with input color and track the position of the cloth where it placed in the rack. The computer pass the location to Arduino Uno, the Arduino Uno move the arm to pick the cloth from that position. 
International Journal of Advanced Research in Computer and Communication Engineering

Vol. 8, Issue 4, April 2019

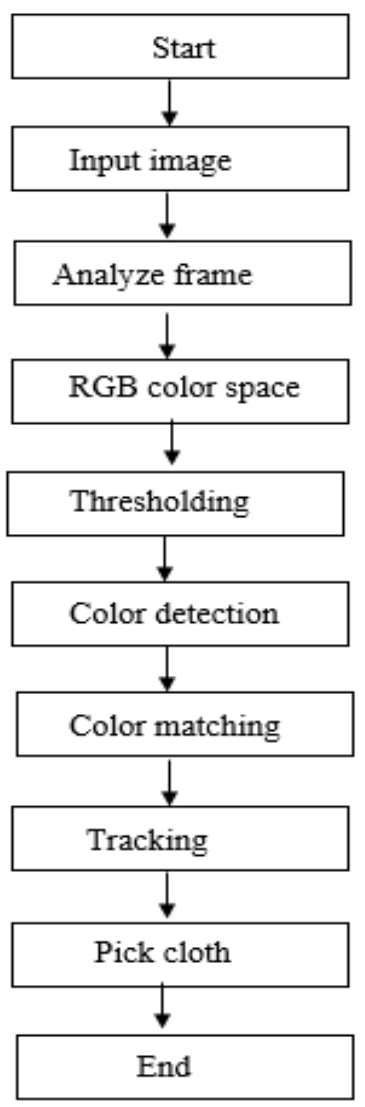

Fig 1: flow chart

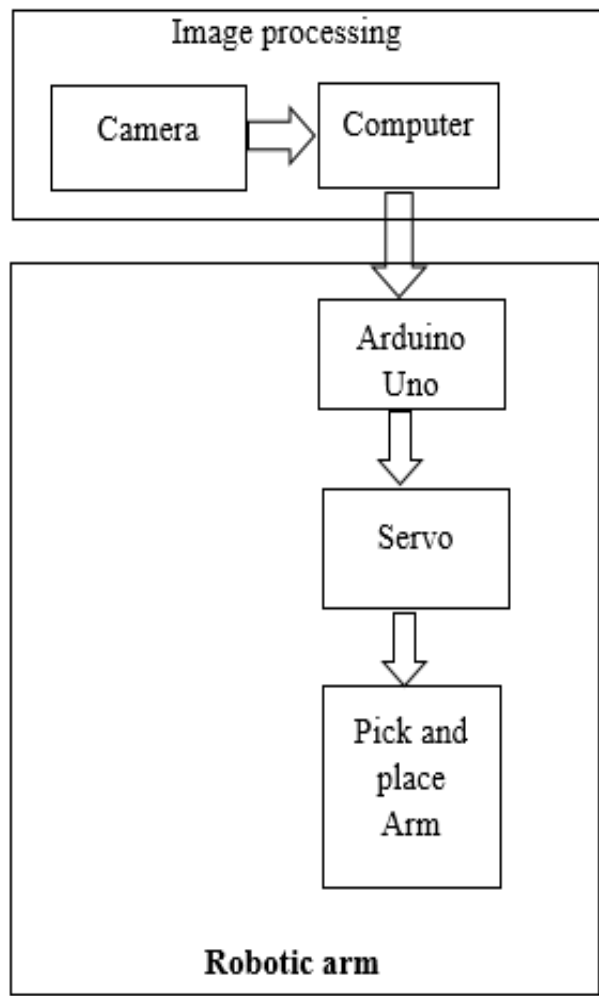

Fig-2: System Block Diagram 


\section{METHODOLOGY}

The system mainly consists of two parts, one is robotic arm and other is image processing.

\subsection{Robotic arm}

A proper and stable structure is essential for carrying the total weight and operating smoothly. Proper design of this structure can efficiently conduct the total work.

Base: This arm is attached with the base. Two servomotors are used here. The first servomotor is capable of rotating 0 degree to 180 degrees. The second servomotor is capable of rotating 180 degrees to 0 degree downward. The motor can push the arm towards down very quickly as the net pressure is downward.

Main base: If the weight of the arm is much heavier, it cannot take the total weight of the arm. The focus of this project is to carry the total weight of the arm. The shoulder is attached with the base by calculating the torque.

Elbow: Two servomotors are used on both sides of the elbow. The size of the servomotor is $3.8 \mathrm{~cm}$ but the space is cut in a size of $3.9 \mathrm{~cm}$ so that it can strongly grip and hold two servomotors.

Wrist: The gripper is attached with it. The main weight of the arm generally depends on this part. The arm length from the base to the shoulder 8.5 inch. Moreover, the second arm elbow length is 6.9 inch. The third arm, i.e., the gripper is 3.9 inch long. So, the total arm length is 19.3 inch. We have used these measurements because if we had used a longer arm, the weight would have been much heavier.

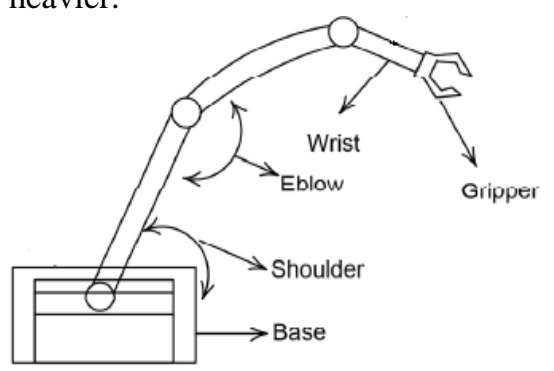

Fig-3: Robotic Arm Structures

Servo motor: Four servomotor are used for arm movement. A servomotor is a rotating actuator or linear actuator that takes into consideration exact control of angular or linear position, speed and acceleration. Servomotors are not a particular class of motors in spite of the fact that the term servomotor is regularly utilized to allude to a motor appropriate for use in closed-loop control system.

Arduino Uno: The Arduino Uno is a microcontroller board based on the ATmega328.The board used for programming the microcontroller in our prototype is Arduino Uno. It has 14 digital input/output pins of which 6 can be used as PWM Outputs, 6 analog inputs, a $16 \mathrm{MHz}$ ceramic resonator USB Connection, a power jack, an ICSP header, and a reset button. It contains everything needed to support the microcontroller; simply connect it to a computer with a USB cable or power it with an AC-to-DC adapter or battery to get started. The Uno differs from all preceding boards in that it does not use the FTDI USB-to-serial driver chip. Instead, it features the Atmega16U2 (Atmega8U2 up to version R2) programmed as a USB to serial port converter. Revision 2 of the Uno board has a resistor pulling the 8U2 HWB line to ground, making it easier to put into DFU mode.

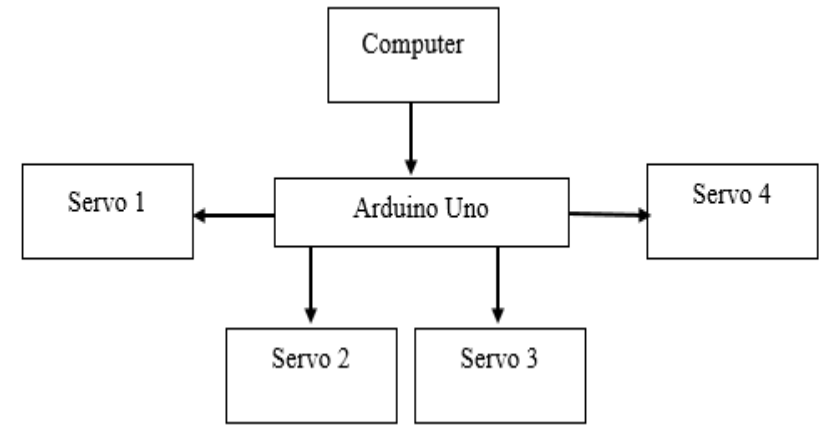

Fig-4: Block Diagram Of Robotic Arm 
Vol. 8, Issue 4, April 2019

\subsection{Image processing}

Digital image processing is the use of computer algorithms to perform image processing on digital images. Digital image processing has many advantages over analog image processing. It allows a much wider range of algorithm to be applied to the input data can avoid problems such as the build-up of noise and signal distortion during processing.

\section{RGB color space}

Because the human eye only has colour sensitive receptors for red, green and blue, it is theoretically possible to decompose every visible colour into combinations of these three "primary colours." Colour monitors, for instance, can display millions of colours simply by mixing different intensities of red, green and blue. It is most common to place the range of intensity for each colour on a scale from 0 to 255 (one byte). The range of intensity is also known as the "color depth". The possibilities for mixing the three primary colors together can be represented as a three dimensional coordinate plane with the values for R (red), G (green) and B (blue) on each axis. This coordinate plane yields a cube called the RGB color space:

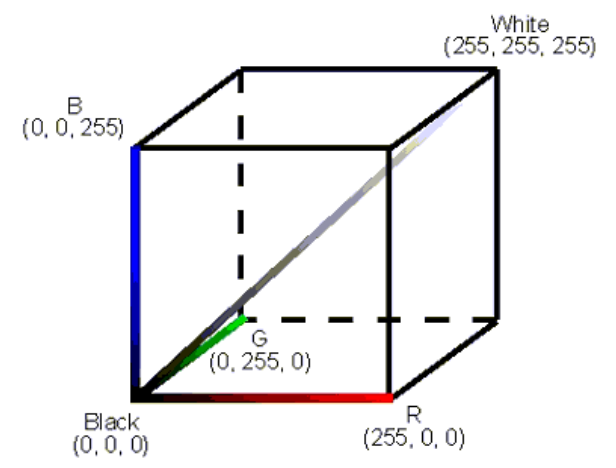

\section{Color detection}

In this system, the clothes color is detected by color detection algorithm using openCV library and matching the clothes by contrast color given by the designer. The camera capture the image of the cloth, analyze the color from the input frame using color thresholding. Detect the color from the input image. In color detection it uses mainly three methods canny (), in range() and contour. The canny () method is used for edge detection, inrange() is used for color thresholding and finally contour used to draw the interested area.

\section{Canny Algorithm}

\section{Steps}

1) Filter out any noise. The Gaussian filter is used for this purpose.

2) Find the intensity gradient of the image. For this, we follow a procedure analogous to Sobel:

a) Apply a pair of convolution masks in $\mathrm{x}$ and ydirections

$$
\begin{aligned}
\mathrm{G}_{\mathrm{x}} & =\left[\begin{array}{ccc}
-1 & 0 & +1 \\
-2 & 0 & +2 \\
-3 & 0 & +3
\end{array}\right] \\
\mathrm{G}_{\mathrm{y}} & =\left[\begin{array}{ccc}
-1 & -2 & -3 \\
0 & 0 & 0 \\
+1 & +2 & +3
\end{array}\right]
\end{aligned}
$$

b) Find the gradient strength and direction with:p

$$
\begin{gathered}
\text { Edge_Grdient, }(G)=\sqrt{G x^{2}+G y^{2}} \\
\text { Angle }(\Theta)=\tan ^{-1}\left(\frac{G y}{G x}\right)
\end{gathered}
$$

c) Non-maximum suppression is applied. This removes pixels that are not considered to be part of an edge. Hence, only thin lines (candidate edges) will remain.

d)Hysteresis: The final step. Canny does use two thresholds (upper and lower):

1. If a pixel gradient is higher than the upper threshold, the pixel is accepted as an edge

2. If a pixel gradient value is below the lower threshold, then it is rejected.

3. If the pixel gradient is between the two thresholds, then it will be accepted only if it is connected to a pixel that is above the upper threshold.

Canny recommended a upper:lower ratio between 2:1 and 3:1. 
Vol. 8, Issue 4, April 2019

\section{Contour}

Contours can be explained simply as a curve joining all the continuous points (along the boundary), having same color or intensity. The contours are a useful tool for shape analysis and object detection and recognition. For better accuracy, use binary images. So before finding contours, apply threshold or canny edge detection. Since OpenCV 3.2, findContours () no longer modifies the source image but returns a modified image as the first of three return parameters. In OpenCV, finding contours is like finding white object from black background. So remember, object to be found should be white and background should be black. To draw the contours, cv.drawContours function is used. It can also be used to draw any shape provided you have its boundary points. Its first argument is source image, second argument is the contours which should be passed as a Python list, third argument is index of contours (useful when drawing individual contour. To draw all contours, pass -1) and remaining arguments are color, thickness etc.

\section{inRange()}

Perform basic thresholding operations using openCV cv::inRnge function.

For example the above red color is detected by range provided in the python coding and RGB image is threshold to get the exact blue color is matched.

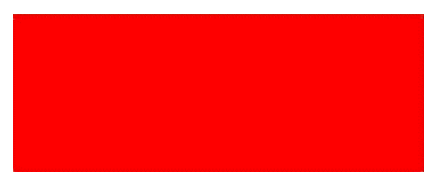

The detected colour is red.

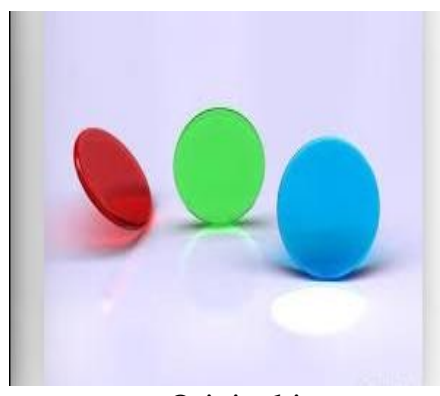

Original image

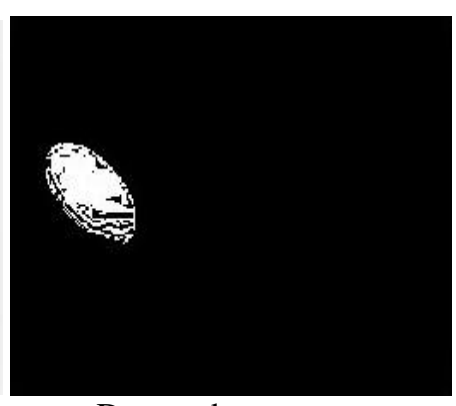

Detected area

Fig-5: Color detection

\section{Color matching and tracking}

A camera placed in front of the rack which contains the cloth. The customer shows the color of the cloth to the robotic system, the camera on the arm capture the image, analyse the color from the frame using color thresholding. Second camera capture the picture and analyse the color .if it matches with input color then track the position of the cloth where it placed in the rack.

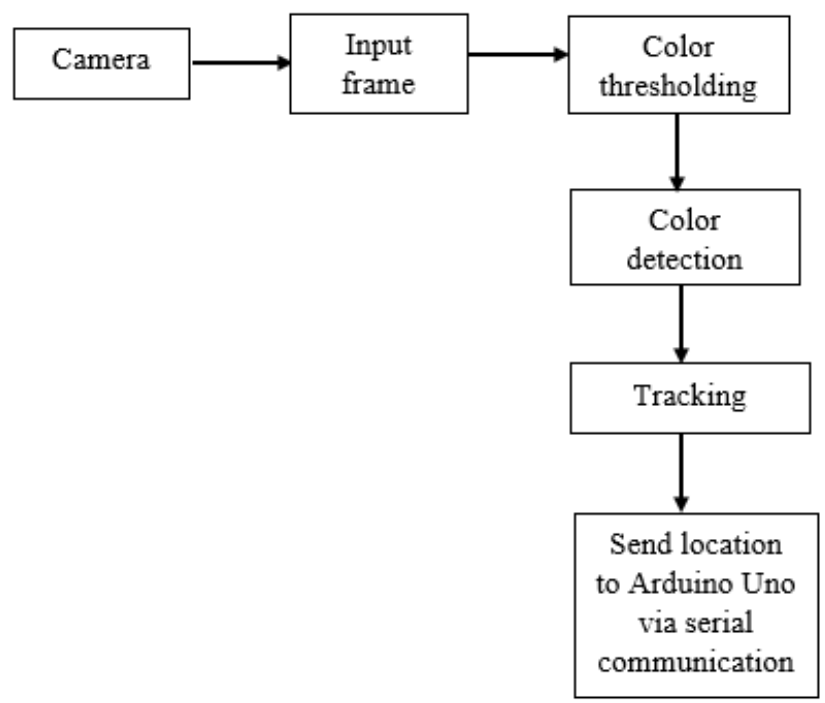

Fig-6: Image Processing 


\section{SIMULATION RESULTS}

We have proposed in our system to recognize clothing colors to help the people. Our proposed system uses the detection of the colors and matching it gives some new approach. Here we are using OPENCV techniques and methods for our system. As an object comes within the viewing angle of the camera, the camera detects the color and takes that as a color input. Then it passes the data to the microcontroller. All the motors follows same forward and backward direction as we preset. The arm picks up object from the picking position and place the cloth in another position. All the work is done by camera and servomotor accurately.

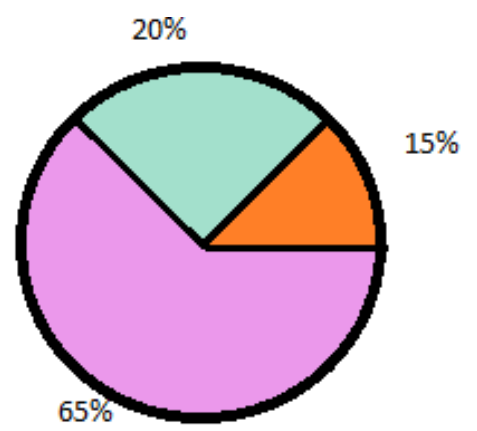

Proposed system

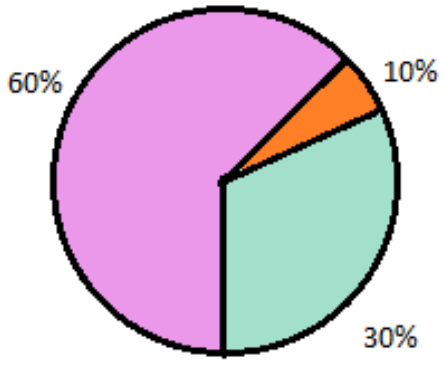

Existing system

Fig-7: Result comparative study

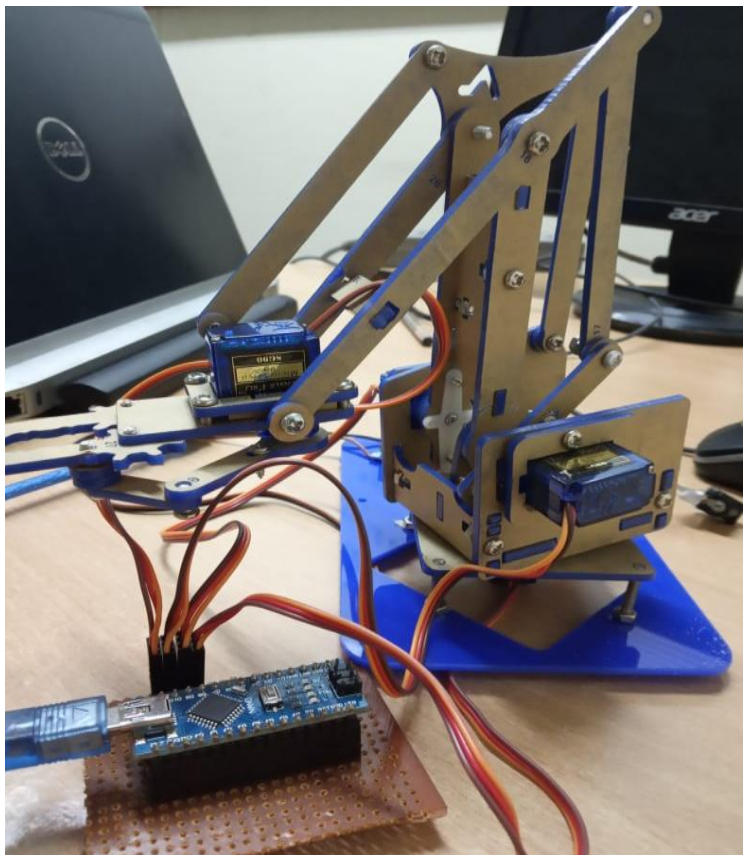

Fig-8: Robotic Arm

\section{CONCLUSION AND FUTURE WORK}

We have successfully designed a multi functioning robot, which consists of moving base and arm with joints with different moving axes that can pick up cloths of different colors. Since this machine does not suffer from human fatigue, it can continuously perform the job of picking cloth according to match of color without any mistake. This system will add accuracy and perfection in solving the problem of picking up matching cloth from rack in textile shop as per customer needs. It will reduce the time needed for selecting cloths from textile shop. It will also reduce the labor cost hence reduce the production cost as well. As a result, textile shop will be able to supply products at a lower cost, which will be beneficial to both the producer and the consumer.

Currently, the robotic sorter can identify only three colors. We plan to improve it so it can identify more colors. The system not identify the patterns so we plan to include the pattern detection also. 


\author{
Vol. 8, Issue 4, April 2019
}

\title{
REFERENCES
}

[1]. S.Prasanna, N.Priyadharshini and M.Arulb Pugazhendhi, "Textile Robot for Matching and Pick Up Clothes Based on Color Recognition " Asian Journal of Applied Science and Technology (AJAST), Volume 1, Issue 3, Pages 62-65, April 2017.

[2]. Md. Jamilur Rahman, Deb Prosad Das, Ohidul Islam, Hasan U. Zaman, “ A Novel Design of a Robotic Object Sorter Based on Color Differences using Image Processing Techniques",February 2018

[3]. Masoud Nosrati,Ronak Karimi ,Mehdi Hariri Kamran Malekian, Edge Detection Techniques in Processing Digital Images: Investigation of Canny Algorithm and Gabor Method,World Applied Programming, Vol (3), Issue (3),ISSN: 2222-2510, March 2013. $116-121$.

[4]. Shuai Yuana, YingLi Tiana*, and Aries Arditi ,"Clothing Matching for Visually Impaired Persons", Research Gate publications,2014 august, Vol. 23, 2011

[5]. Krishna Kumar Pandey, Nishchol Mishra,Design \& development of color matching algorithm for image retrieval using histogram and segmentation techniques, International Journal of Information Technology and Knowledge Management, July-December 2011, Volume 4, No. 2, pp. 585-589.

[6]. Mr.Jeeva B, Sanjay V, Vikram Purohit, Derryl Oswald Tauro, Vinay J "Design and Development of Automated Intelligent Robot Using OpenCV “,2018 International Conference on Design Innovations for 3Cs Compute Communicate Control(ICDI3C) ,August 2018

[7]. S.Prasanna, N.Priyadharshini and M.Arul Pugazhendhi, Android Application to Assist Visually Impaired with Outfit Coordination " International Journal of Computer Applications (0975 - 8887) Volume 108 - No 19,December 2014

[8]. Jarin Joe Rini J, Thliagavathi B," Recognizing clothes patterns and clours for blind people using neural network",IEEE,2015

[9]. Thogaricheti Ashwini1, Mrs. Anuradha.S., “Assistive clothing Pattern Recognition for Impaired people”

[10]. International Journal of Advanced Research in Computer and Communication Engineering Vol. 5, Issue4, April 2016.

[11]. Ester Hsu,Christie Paz,Shizhe Shen, "Clothing Image Retrieval for Smarter Shopping EE368,Standord University Stanford California 94305.

[12]. L. Dhamini, and D. Bhanuprakash, "Color Recognition System for Visually Impaired Persons “, CVR Journal of Science and Technology, Volume 14, ISSN 2277 - 3916, June 2018

[13]. Krishna Kumar Pandey, Nishchol Mishra,Design \& development of color matching algorithm for image retrieval using histogram and segmentation techniques, International Journal of Information Technology and Knowledge Management, July-December 2011, Volume 4, No. 2, pp. 585-589.

[14]. V. Subbarayudu, D. Vishnu Vardhan "Efficient Clothing Pattern Recognition for Blind People Using SVM Classifier", International Journal of Science and Research (IJSR) ISSN (Online):2319-7064,Volume 4 Issue 7, July 2015.

[15]. Ibrahim Patel, Jagdish Goud "Color Recognition for Blindand Color Blind People". International Journal of Engineering and Innovative Technology (IJEIT) Volume 2, Issue 6, December 2012

[16]. OpenCV, [Online]. Available: http://opencv.org/. 